\title{
Localização de Robôs Móveis em Ambiente Internos usando Marcos Fiduciais
}

\author{
Gabriel da Silva Santos; Etevaldo Cardoso²; Marco Antonio dos Reis ${ }^{2}$ \\ ${ }^{1}$ SENAI CIMATEC; Salvador/Ba; aec.gabrielsantos@gmail.com \\ ${ }^{2}$ SENAI CIMATEC; Salvador/Ba ;
}

Resumo: O trabalho teve por objetivo utilizar marcos fiduciais para localizar um robô em ambientes internos utilizando simulação como ferramenta de testes. Foi desenvolvido um ambiente com marcos fiduciais espalhados para o robô identificar e poder recalcular a sua posição comparado com o referencial dos sensores de odometria. Foi possível observar que este método aplicado para ambientes internos tem um baixo custo computacional, causado pela facilidade de leitura dos marcos, e uma alta precisão na localização do robô em comparação com outros métodos.

Palavras-Chave: Localização; Marcos fiduciais; Simulação;

\section{Indoor localization for Mobile Robots using Fiducial Markers}

\begin{abstract}
The work aimed to use fiducial markers to locate a robot indoors using a simulation environment as a testing tool. An environment with scattered fiducial markers was developed for the robot to identify and be able to recalculate its position with the odometer sensors reference frame. It was observed that this method applied for indoor environments has a low computational cost, caused for the readability of the landmarks, and a high accuracy in robot localization compared to other methods.
\end{abstract}

Keywords: Localization; Fiducial markers; Simulation; 


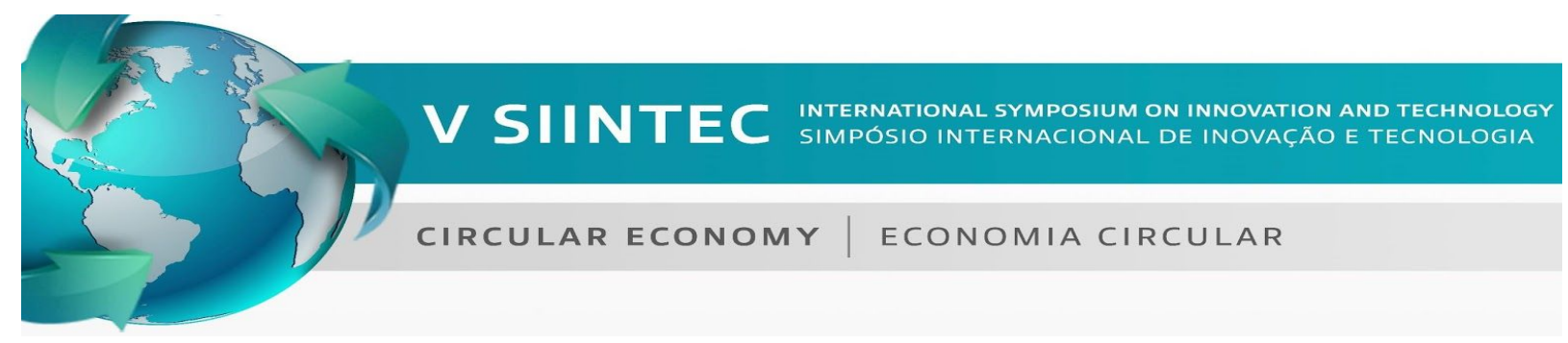

\section{INTRODUÇÃO}

Com o advento de técnicas mais aprimoradas, sensores mais precisos e maior poder de processamento os robôs móveis tem tido um aumento de possíveis aplicações nas quais eles podem ser utilizados. Para tal eles precisam realizar atividades que incluem movimentação e tomada de decisão, mas para isso eles precisam de informações dos seus sensores que permitam a eles uma noção precisa de onde eles estão para poderem iniciar as suas atividades com precisão e por isso a localização é um elemento vital para o desenvolvimento da robótica móvel.

\subsection{Localização e sensores}

Localização é o lugar em que algo se encontra e o ato ou efeito de localizar. Dentro da robótica móvel a localização é uma informação indispensável, pois indica ao robô em que parte do ambiente ele está e a partir disso ele pode tomar as decisões cabíveis a cada situação.

Por se tratar de uma informação primordial para os processos foram desenvolvidas diversas técnicas de localização como Filtro de Bayes, Método de Markov, Filtros Gaussianos, Filtros de Particulas[1]. Para que cada um desses métodos possam ser aplicados é necessário extrair as informações do ambiente através de sensores que podem ser encoders, IMUs (Inertial measurement unit), GPS (Global Positioning System), LIDAR (Light Detection And Ranging) e câmeras[2].

Cada sensor extrai tipos distintos de informações, mas todos com o objetivo de fornecer conhecimento sobre o ambiente em que o robô se encontra. No caso de encoders e IMUs a informação que o robô recebe é referente a sua variação dentro do ambiente, onde a IMU entrega os valores de aceleração angular [3] e o enconder o quanto o robô movimentou-se em relação ao ponto inicial. O LIDAR é um sensor óptico que mede as propriedades da luz refletida a fim de obter a distância ou outras informações de um objeto distante [4]. As câmeras são sensores que extraem as informações necessárias para o reconhecimento da posição através do processamento das imagem, que por sua vez podem ter ou não elementos com a função de demarcar um espaço no ambiente, como os marcos fiduciais artificiais.

\subsection{Marcos Fiduciais}

Marcos fiduciais são objetos no campo de visão de um sistema que são utilizados como pontos de referência ou medida. Eles são utilizados em diversas áreas, como na medicina no tratamento contra o câncer onde eles são utilizados como marcadores na radioterapia para facilitar os alvos do tratamento contra um tumor, já na microeletrônica são utilizados na construção de placas de circuitos impressos e são alocados para localizar as partes da placa. 


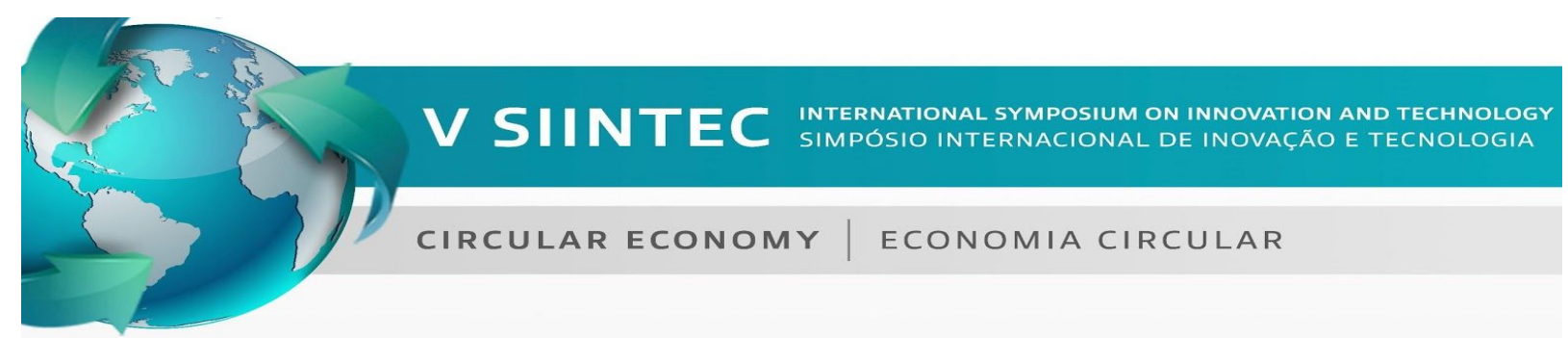

$\mathrm{Na}$ robótica são utilizados os marcos fiduciais artificiais, que são padrões gerados para serem interpretados por um sistema de visão computacional. Esses marcos tem a capacidade de apresentar informações que variam de acordo com o seu padrão e são aplicados quando velocidade e precisão são requeridos pelo sistema, pois são fáceis de serem detectados [5].

Entre os diversos tipos de marcos fiduciais que existem os que são baseados em superfícies quadráticas se tornaram muito atraentes por permitirem a extração da posição da câmera através das quatro quinas, como é possível observar na Figura 1.

Figura 1. Exemplos de marcos fiduciais artificiais.

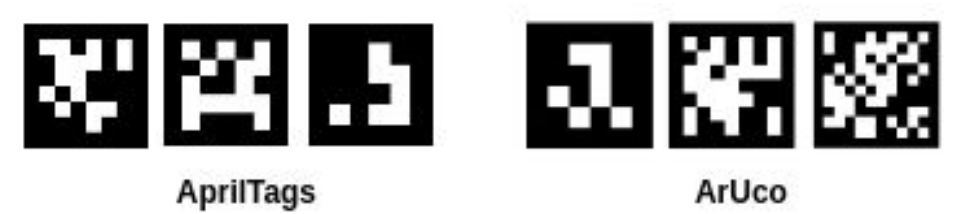

\subsection{Posição e Orientação utilizando um Marco Fiducial}

A estimativa da posição e orientação de uma câmera calibrada utilizando pontos no mundo e projeções em uma imagem é um problema chamado de Perspective-n-Point. Para este estudo, é utilizada a função solvepnp disponível na biblioteca do OpenCV (Biblioteca de Visão Computacional), onde é possível fornecer a posição na imagem em pixels dos contornos dos ArUcos, previamente detectados, e então retornar com os valores de translação e rotação em relação a câmera[6][7][8], como está representado na Figura 2.

Figura 2. Extração do posicionamento através da câmera.

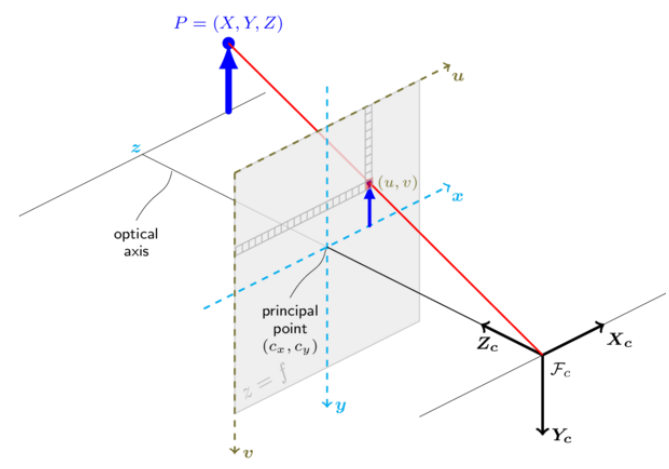

\section{METODOLOGIA}

Os experimentos deste trabalho foram feitos em um ambiente de simulação no Gazebo. Ele é um software de código aberto mantido pela OSRF (Open Source Robotics Foundation) que possibilita a simular as interações do robô com um ambiente físico fielmente. Além disso o Gazebo possui integração com o ROS (Robot Operating System), que é o framework utilizado para o desenvolvimento do trabalho. 


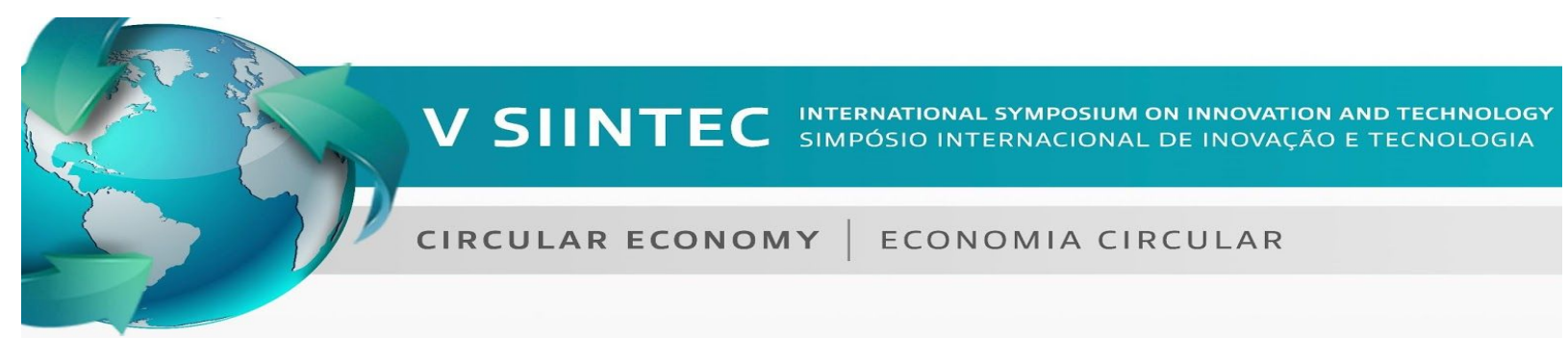

A câmera utilizada na simulação foi uma câmera de simulação genérica, com resolução 640x480px, com a matriz de calibração da câmera dada por (1), quanto aos marcos fiduciais, o padrão escolhido foi ArUco[5][9], pois possuem extensas bibliotecas, utilizadores e estudos. Nos experimentos foi utilizada ArUcos do dicionário $6 \times 6$ com dimensões de $100 \mathrm{~mm}$ e $150 \mathrm{~mm}$. O ambiente da simulação foi uma área de $48 \mathrm{~m}^{2}$.

$$
K=\left[\begin{array}{ccc}
530.47 & 0.00 & 320.50 \\
0.00 & 530.47 & 240.50 \\
0.00 & 0.00 & 1.00
\end{array}\right]
$$

O robô utilizado para os testes na simulação foi o TURTLEBOT3 Waffle PI da empresa ROBOTIS com dimensões de comprimento, largura e altura de $281 \mathrm{~mm} x$ $306 \mathrm{~mm} \times 141 \mathrm{~mm}$ respectivamente, com peso de $1.8 \mathrm{~kg}$, representado na Figura 3.

Figura 3. Robô no ambiente do Gazebo.

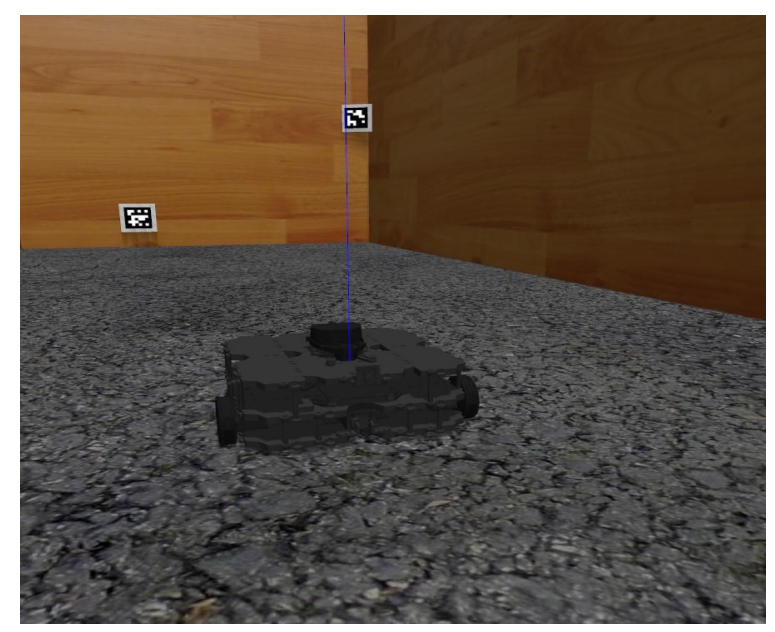

Para a análise dos dados da posição da base do robô, não foi utilizado nenhum filtro para fusão de medidas com a odometria, permitindo a possibilidade de visualizar os dados diretamente da inferência do algoritmo de cálculo de posicionamento do robô.

Foram realizados experimentos, seguindo o fluxograma na Figura 4, posicionando o robô no mundo da simulação, onde o objetivo foi estimar a posição e comparar com a posição "real"do robô fornecida pelo simulador. Os experimentos se diferenciam pela orientação dos marcadores em relação ao mundo, e com a movimentação ou não do robô durante as tomadas de imagens para o cálculo da posição.

Figura 4. Fluxograma de Simulação e Análise dos dados. 


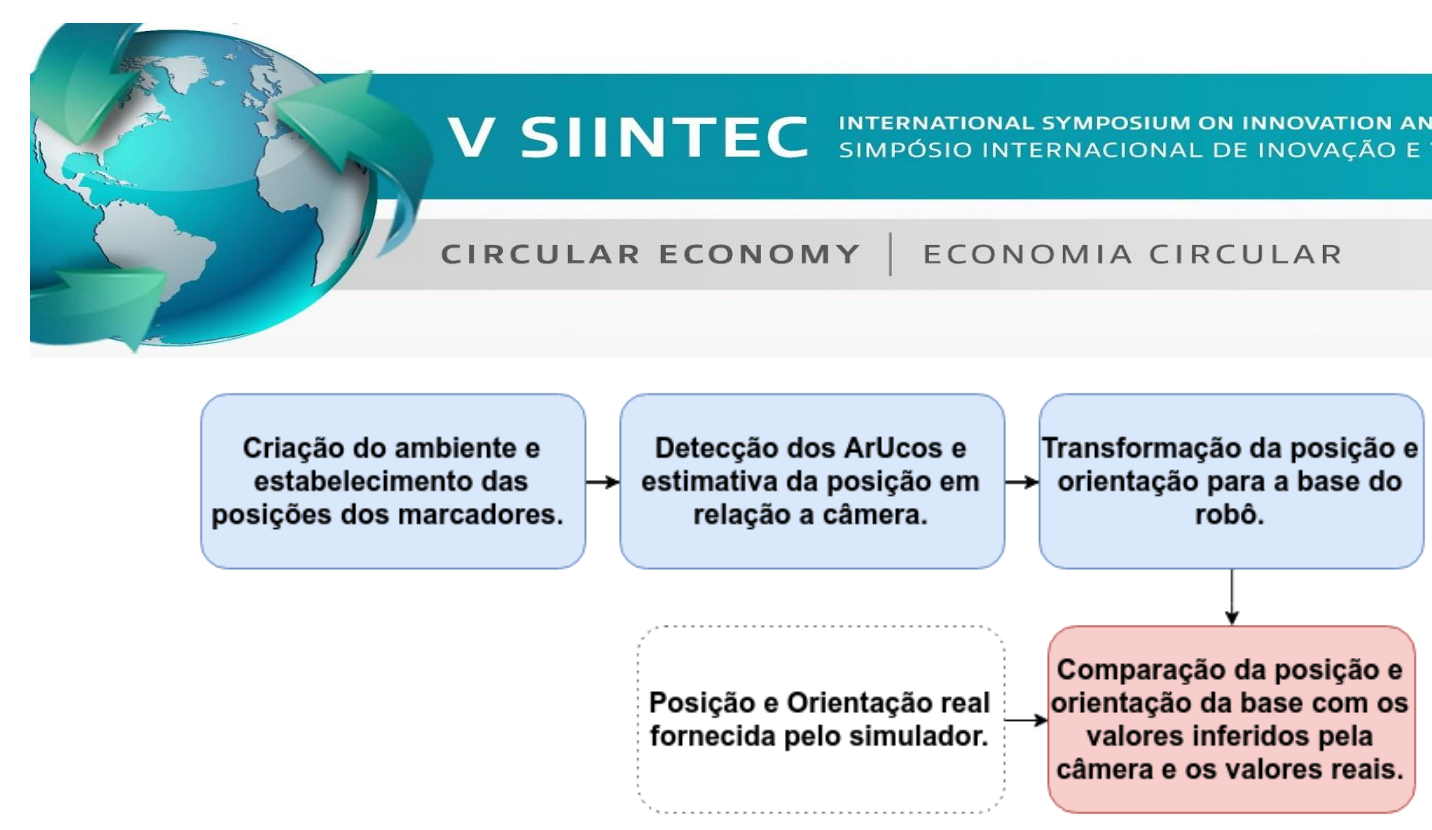

\section{RESULTADOS E DISCUSSÃO}

\subsection{Média de Posição e Quatérnios}

Com o objetivo de garantir uma qualidade maior na obtenção das posições dos Marcos Fiduciais em relação ao frame ótico da câmera, foi calculada uma média da pose completa. Para o cálculo da média da pose, foi obtido $\mathrm{N}$ amostras para cada medida, e para cada amostra se obtém a matriz de posição e matriz de quatérnios. Para a média da posição foi utilizada a equação (2), onde At é área total do ArUco na imagem (em px), Ai é a área de cada ArUco. pi é a posição de cada marco e $\mathrm{p}^{-}$é a posição média.

$$
\left[\begin{array}{l}
\bar{p}_{x} \\
\bar{p}_{y} \\
\bar{p}_{z}
\end{array}\right]=\frac{1}{A_{t}} \sum_{i=0}^{N} A_{i}\left[\begin{array}{l}
p_{x} \\
p_{y} \\
p_{z}
\end{array}\right]_{i}
$$

Foi utilizado o recurso da área, pois tendo uma área maior o marco se torna usualmente mais confiável[6], logo, é interessante inserir esse parâmetro para diminuir o peso de marcos menores ou que estejam mais distantes. Por motivos análogos a média da posição, foi necessário calcular a média dos quatérnions, responsáveis pela orientação do robô, utilizado o método de Markley[10].

$$
Q=\frac{1}{A_{t}} \sum_{i=0}^{N} A_{i} q_{i} q_{i}^{t} \quad Q v=\lambda v
$$

\subsection{Transformações}

As transformadas homogêneas são transformadas que descrevem a posição e orientação de algo em relação à um dado referencial. O objetivo principal deste estudo foi obter a transformada entre a base do robô e a referência global (W Tbase), representada na Figura 5.

Figura 5. Representação das transformadas 


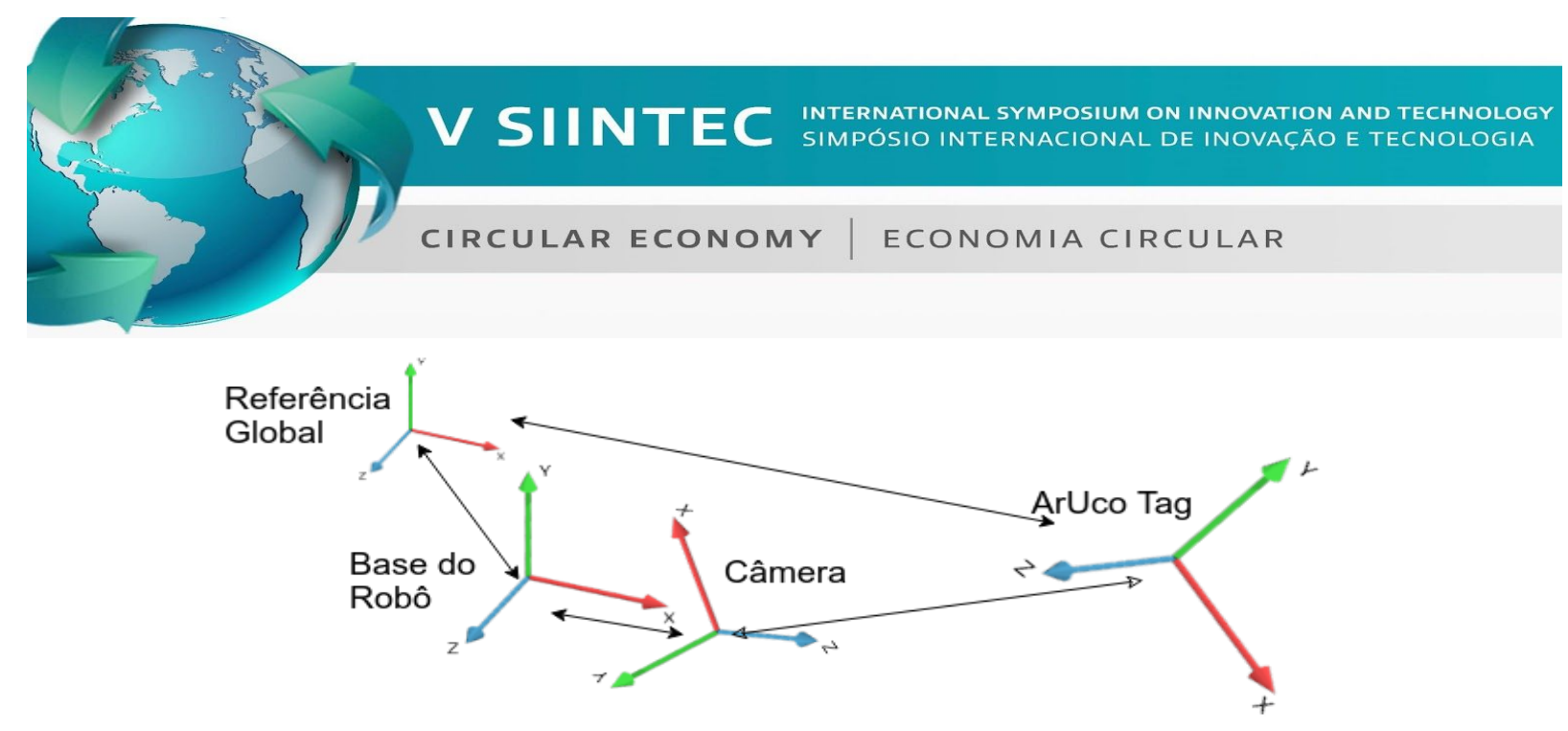

Para conseguir isso, foi utilizada as transformadas predefinidas dos marcos com relação a referência global ( ${ }^{\mathrm{W}} \mathrm{T}_{\text {tag }}$ ) e da câmera para a base do robô (base $\mathrm{T}_{\text {cam }}$ ). Para obter a transformada desejada, foi utilizada a equação (4), onde o único elemento desconhecido até então é ${ }^{\text {tag }} \mathrm{T}_{\text {cam }}$, que é obtido utilizando os ArUcos.

$$
{ }^{W} T_{\text {base }}={ }^{W} T_{\text {tag }} \times{ }^{\text {tag }} T_{\text {cam }} \times{ }^{\text {base }} T_{\text {cam }}^{-1}
$$

É importante relembrar a equação (4) em sua forma completo é dado pela equação (5), porém $\vee$ que representa o ruído das medições foi descartado dos cálculos por motivos de simplificação.

$$
{ }^{W} T_{\text {base }}={ }^{W} T_{\text {tag }} \times{ }^{\text {tag }} T_{\text {cam }} \times{ }^{\text {base }} T_{\text {cam }}^{-1}+V
$$

\subsection{Simulações}

Para as simulações, foi desenvolvido duas baterias de experimentos, uma com o robô estático no ambiente, e outro com o robô em movimento. Dessa maneira é possível diferenciar a eficiência da medição quando ocorrem mudanças bruscas na imagem. Foram escolhidos 4 pontos aleatórios no ambiente de simulação onde o robô poderia visualizar no mínimo 2 marcos dispostos no ambiente. Com esses quatro pontos o robô inicializou a câmera e utilizando a média da posição obtida a partir dos marcos ArUcos calculou a transformação ${ }^{\text {tag }} \mathrm{T}_{\text {cam }}$, e então foi utilizada a equação (4) para encontrar a relação do robô com o referencial global. A Figura 6 exibe as posições "reais" do robô, com a legenda Real(), e as posições inferidas, com a legenda Cal(). Os círculos representam a localização do robô no mapa, enquanto os traços representam a orientação.

Já os testes realizados com o robô em movimento tiveram os resultados das posições "reais" e os inferidos comparados separadamente com seus correspondentes. Alguns momentos a posição foi alterada bruscamente para visualização do efeito na inferência da medição, porém apesar de um desvio, o resultado converge para o resultado esperado, apresentado na Figura 7.

Figura 6. Posições Estáticas. 


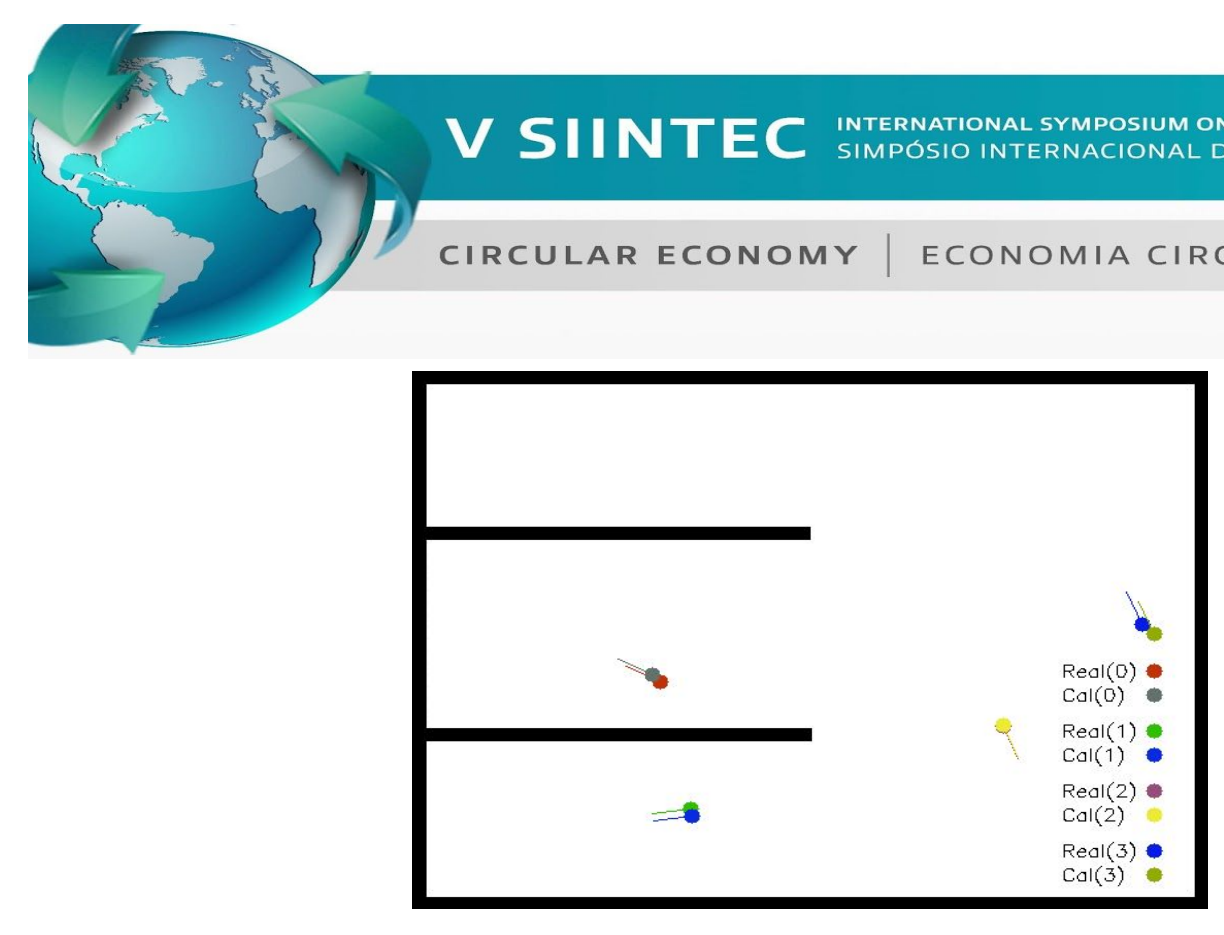

Figura 7. Comparação entre variáveis inferidas e reais.

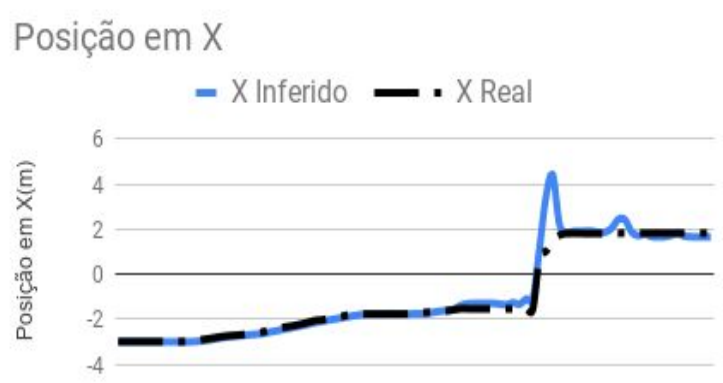
Posição em Y
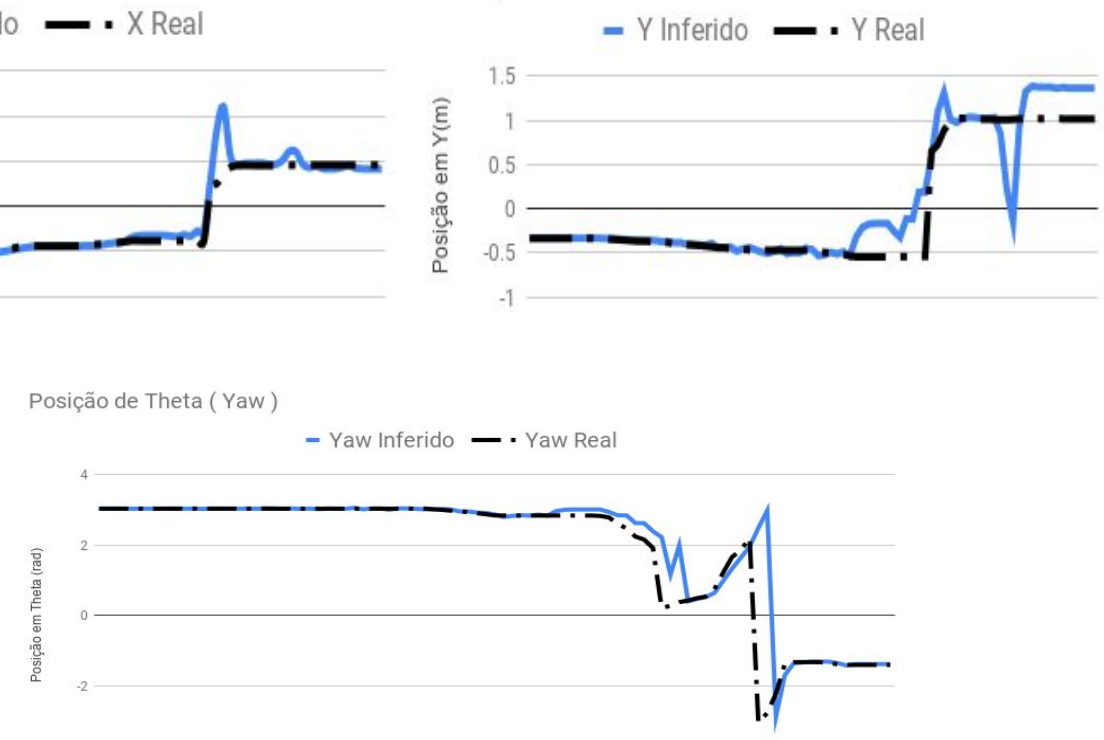

É possível perceber que em momentos onde a posição é variada lentamente o erro associado a posição inferida se torna baixo, porém quando o marco tem sua posição variada de forma brusca, em relação a câmera, o erro é aumentado.

\section{CONCLUSÃO}

As figuras 6 e 7 demonstram que é possível realizar a localização com alto grau de confiança, porém em algumas condições podem não possuir um desvio que deve ser considerado. Todavia a inclusão de filtros preditivos e fusão sensorial podem diminuir a imprecisão. O que abre oportunidades para novos estudos visando entender como e quanto pode ser aprimorado a técnica de localização. 


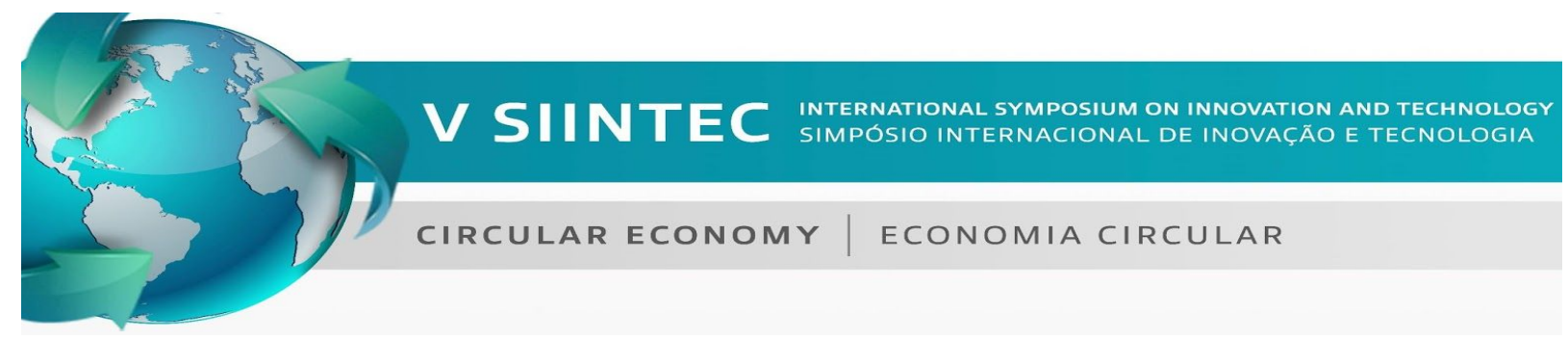

Apesar de possuir erros associados a sua medição, os marcos fiduciais se mostram muito úteis por utilizarem um algoritmo de baixo custo computacional e sendo necessário somente uma câmera, com isso, pode ser interessante sua implementação em aplicações às quais esses erros se tornem aceitáveis ou desprezíveis e também para fornecer uma confiança na localização global no processo de navegação utilizando outras técnicas como o SLAM (Simultaneous Localization and Mapping).

\section{REFERÊNCIAS}

[1] Sebastian Thrun, Wolfram Burgard, and Dieter Fox. Probabilistic robotics. MIT press, 2005.

[2] Maja J Mataric, Humberto Ferasoli Filho, José Reinaldo Silva, and Silas Franco dos Reis Alves. Introdução à robótica. 2014.

[3] AD King. Inertial navigation-forty years of evolution. GEC review, 13(3):140-149, 1998.

[4] Marcos Giongo, Henrique Soares Koehler, Sebastião do Amaral Machado, Flavio Felipe Kirchner, and Marco Marchetti. LiDAR: princípios e aplicações florestais. Pesquisa Florestal Brasileira, 30(63):231, 2010.

[5] Francisco J Romero-Ramirez, Rafael Muñoz-Salinas, and Rafael MedinaCarnicer. Speeded up detection of squared fiducial markers. Image and vision Computing, 76:38-47, 2018.

[6] Alberto López-Cerón and José M Canas. Accuracy analysis of marker-based 3D visual localization. In XXXVII Jornadas de Automatica Workshop, 2016.

[7] Radu Horaud, Bernard Conio, Olivier Leboulleux, and Bernard Lacolle. An analytic solution for the perspective 4-point problem. In Proceedings CVPR'89: IEEE Computer Society Conference on Computer Vision and Pattern Recognition, pages 500-507. IEEE, 1989.

[8] Andrej Babinec, Ladislav Jurišica, Peter Hubinsky, and František Ducho ' n.” Visual localization of mobile robot using artificial markers. Procedia Engineering, 96:1-9, 2014.

[9] Sergio Garrido-Jurado, Rafael Muñoz-Salinas, Francisco José Madrid-Cuevas, and Manuel Jesús Marín-Jiménez. Automatic generation and detection of highly reliable fiducial markers under occlusion. Pattern Recognition, 47(6):2280-2292, 2014.

[10] F Landis Markley, Yang Cheng, John Lucas Crassidis, and Yaakov Oshman. Averaging quaternions. Journal of Guidance, Control, and Dynamics, 30(4):11931197, 2007. 